\title{
PHYTOHORMONES - METABOLIC BY-PRODUCTS OF MICROORGANISMS. IDENTIFICATION METHODS.
}

\section{Dimova S. B.}

Institute of Agricultural Microbiology and AgroIndustrial Production NAAS, 97, Shevchenko str., Chernihiv, 14027, Ukraine

E-mail: dimova13@yandex.ua

The paper presents the overview of scientific publications relating to the modern ideas on plant hormones biosynthesis by microorganisms. Author describes advantages and disadvantages of existing analytical methods used for identification of phytohormonal content in microbiological studies.

Keywords: phytohormones, auxins, cytokinins, gibberellins, microbial synthesis of phytohormones, methods of plant hormones identification.

The uprising interest to phytohormones is driven by natural origin of plant hormones along with their consistent use by humanity that eliminate the uncertainty of their aftereffects [1]. The available to date scientific knowledge of plant hormones is important when solving practical problems in agricultural production due to the essential role of hormonal system in regulation of ontogenesis of plants $[2,3]$.

Charles Darwin pointed out the existence of plant substances functionally similar to the hormones of animals for the first time in 1880 in his paper "Power of movement of plants" [4]. In the 30 years of the twentieth century, a hormone responsible for reactions observed by Darwin was isolated and identified as indolyl-3-acetic acid (IAA). Many Ukrainian and Russian scientists were studying the effect of phytohormones during the last century, despite the fact that the prospects in this area were repeatedly questioned. Talking about the formation of this area in biological science the founders of phytohormonology in Ukraine should be mentioned and among them are Kholodny M.G. (Institute of Botany and Taras Shevchenko National University of Kyiv) [5] Chailakhyan M.Kh.[6, 7] (Institute of Plant Physiology RAS) and researchers of this institute, under the supervision of Kulayeva O.M. [8 - 10], Polevoy V.V. and his colleagues [2] (St. Petersburg State University), Kudoyarova G.R. and Veselov S.Yu. [11, 12] - scientists who are now successfully work at the Institute of Biology, Ufa Scientific Center of RAS. Fundamental research of plant hormones is also continues in Ukraine at the Institute of Plant Physiology and Genetics, National Academy of Sciences, Zabolotniy D.K. Institute of Microbiology and Virology, National Academy of Sciences, Institute of Agricultural Microbiology and Agroidustrial Production NAAS.

Nowadays, compounds related to the plant hormones are described as regulatory compounds synthesized in plants in small amounts, transported and induce growth and morphological processes in plants. Overall the compound is referred to phytohormones, if it has the following characteristics:

- Induce specific physiological response (plant hormones trigger large development 
programs not only at the cell level, but also at the tissues, organ and plant levels);

- Practically is not enrolled in cell metabolism and is only used for signaling functions;

- Is synthesized in plants by specific group of cells and aimed to affect the another group (the sites of synthesis and action are different, which means that signal compound should be transported); at this any plant cell is capable of the synthesis of plant hormones;

- Compounds with low molecular weight (less than $2 \mathrm{kDa}$ );

- Operates at extremely low concentrations $-10^{-5}-10^{-12} \mathrm{~mol} / 1$.

Most of these properties are relevant to the compounds that are traditionally considered as plant hormones and referred to the five main groups of phytohormones: auxins, cytokinins, gibberellins, abscisins and ethylene. Each group performs typical functions similar in different plant species. Recently in addition to the five "classic" groups of phytohormones brassinosteroids, oligosaccharides, salicylic and jasmonic acids, some phenolic compounds and some other substances that meet the criteria to phytohormonal substances were discovered in plants [13, 14]. However, some of the classical properties are not specific to all plant hormones. Thus, the exceptions are:

- Abscisins, since they are usually act at the point of synthesis, transporting only over short distances;

- Ethylene is transported only in form of its predecessor;

- Phenols, salicylic and jasmonic acids are operating in concentrations greater than $10^{-5} \mathrm{~mol} / 1$.

In general, there is no such process in plant organism, which would not be affected by plant hormones. Their action is observed throughout the entire plant ontogenesis - from seed germination until late plant maturing. Obviously, over time, the list of plant growth regulators will grow and it will enhance our understanding of how hormonal system regulates plants' ontogenesis.

Among plant hormones the growth stimulators and inhibitors are distinguished. Nevertheless, dosage play the crucial role in application of phytohormonal substances growth stimulators used in doses exceeding their optimum range can inhibit plant growth [15 - 17]. Correlation of phytohormonal action to the dose (action force) is expressed graphically as curve. Dose-effect curve consists of subliminal zone when the dose is too low to influence the changes, zone of physiological processes stimulation, inhibition and lethal zones. Hormones (as well as mineral nutrients and many other environmental factors) are characterized with a plateau in the middle of the curve. This means that changes in the dose within that range will not substantially influence on its effect. Big segments of the ascending and descending graph sections indicate the presence of direct relation of effect to the logarithm of the phytohormones dose.

Existing knowledge about plant hormones and their mechanisms of action has been successfully used to solve practical problems in agriculture. Nowadays, many plant hormones are identified and synthesized which opens some prospects for controlling plant growth and development in the desired direction. However, there are still lot of questions 
remain unclear which provides huge opportunities for scientists, not only for plant biologists but also for microbiologists that study plant-microbial interactions, since vast amount of plant hormones are produced not only by plants but also by many microorganisms. It should be noted that for a long time phytohormone studies were only performed by plant physiologist as the common notion was that plants produce hormones in adequate quantities.

According to the modern concepts in any agrocoenosis various molecular interactions occur between plants and microorganisms, accompanied by exchange of metabolites. Given the complexity of plant-microbial interactions it should, however, be noted that an important role in this relationship belongs to phytohormones - metabolic products of microorganisms. Microorganisms promote the formation in rhizosphere zone fund of available plant nutrients and biologically active compounds including phytohormones. Thus, hormones act not only as intracellular signals in plants, but also mediate the interaction between plants and soil microorganisms, which are an integral part of any agrocoenosis [18 - 20]. Given the fact that phytohormones are produced not only by plants, but also by many microorganisms (bacteria can produce these substances even in greater quantities than plants), hormonal regulation today considered as one of the fundamental components of the genetic program implementation mechanism of ontogeny and phylogeny of microorganisms and plants [21].

Microorganisms producers of phytohormones can enter into associative and symbiotic relationship with the host plant or lead to the development of its pathogenesis. Bacteria, micromycetos and algae produce plant growth stimulators - phytohormones of auxin, cytokinin and gibberellin nature (Table). It should be noted that quantitative and qualitative composition of plant hormones synthesized by microorganisms is usually strain specific.

Microorganisms are also capable of synthesis other plant hormones or phytohormonal compounds - ethylene, abscisic acid, brassinosteroids, oligosaccharides, salicylic and jasmonic acids [21]. Thus, microorganisms can produce almost each described to date phytohormone, some of which (such as gibberellins and cytokinins) were actually identified in microbial cultures prior to their discovery in in plants [22-24].

Phytohormones produced by microorganisms belong to the secondary metabolites formed during the specific part of chain reactions within the metabolism of their producer. The maximum number of phytohormones is synthesized by microorganisms during the stationary growth phase, at almost depleted medium and dramatic decrease of cell division processes. This suggests that the excretion of plant hormones by bacteria in unfavorable conditions may have important functional role, increasing the probability of association formation with the plant.

In recent publications on agricultural microbiology, there are a lot of works devoted to the study of microorganisms-producers of substances of phytohormonal nature [25-48]. It has been proved that bacteria affect growth and development of plants through the changes in the concentration of phytohormones. Hormone content in any part of the plant is determined by its production (both by plant, soil and endophytic microorganisms), transport, inter conversion of active and inactive forms and its break-up [9]. In order to 
Table. Literature data on the phytohormones production by microorganisms in vitro

\begin{tabular}{|c|c|c|c|}
\hline Microorganisms & $\begin{array}{c}\text { Plant } \\
\text { hormone }\end{array}$ & $\begin{array}{l}\text { Amount in } \\
\text { cultural media }\end{array}$ & Publication \\
\hline $\begin{array}{l}\text { Association of } \\
\text { microorganisms Azotobacter } \\
\text { chroococcum }+ \\
\text { Azospirillum spp. }\end{array}$ & IAA & $39.8-75.4 \mathrm{mg} / 1$ & $\begin{array}{l}\text { Dyagteryova I.A., } \\
2005\end{array}$ \\
\hline Azospirillum brasilense $\mathrm{Sp} 7$ & IAA & $15 \mathrm{mg} / 1$ & Iosinenko A.D., 1992 \\
\hline $\begin{array}{l}\text { Azospirillum brasilense } \\
\text { SR } 80\end{array}$ & IAA & $24 \mu \mathrm{g} / \mathrm{ml}$ & $\begin{array}{l}\text { Bondarenkova A.D., } \\
2009\end{array}$ \\
\hline Azotobacter sp. & $\begin{array}{l}\text { auxins, } \\
\text { cytokinins }\end{array}$ & $14-74 \mu \mathrm{g} / \mathrm{g} \mathrm{CDB} *$ & $\begin{array}{l}\text { Leonova N.O., } \\
\text { Bilyavska L.O., } 2010\end{array}$ \\
\hline \multirow{3}{*}{$\begin{array}{l}\text { Enterobacter nimipressuralis } \\
32-3\end{array}$} & cytokinins & $620 \mu \mathrm{g} / 1$ & \multirow{3}{*}{$\begin{array}{l}\text { Chaikovska L.O., } \\
\text { Baranska M.I., } 2009\end{array}$} \\
\hline & IAA & $449 \mu \mathrm{g} / 1$ & \\
\hline & gibberellins & $18781 \mu \mathrm{g} / 1$ & \\
\hline $\begin{array}{l}\text { Pseudomonas aurantiaca B- } \\
162\end{array}$ & gibberellins & $13.18 \pm 0.34 \mathrm{mg} / \mathrm{ml}$ & \multirow{2}{*}{$\begin{array}{l}\text { Maksimova N.P. et al., } \\
2009\end{array}$} \\
\hline Pseudomonas mendocina & IAA & $13.5 \mathrm{mg} / 1$ & \\
\hline $\begin{array}{l}\text { Pseudomonas aureofaciens } \\
\text { ИБ6 }\end{array}$ & cytokinins & $1150 \mathrm{ng} / \mathrm{ml}$ & $\begin{array}{l}\text { Asabina E.A., } \\
\text { Chetverikov S.P., } \\
2010\end{array}$ \\
\hline Pseudomonas sp. & IAA & $1.55-10.70 \mu \mathrm{g} / \mathrm{ml}$ & \multirow{6}{*}{ Tsevkelova E.A., 2005} \\
\hline Mycobacterium sp. & IAA & $1.52-17.4 \mu \mathrm{g} / \mathrm{ml}$ & \\
\hline Artrobacter sp. & IAA & $1.18 \mu \mathrm{g} / \mathrm{ml}$ & \\
\hline Bacillus sp. & IAA & $2.33-6.30 \mu \mathrm{g} / \mathrm{ml}$ & \\
\hline Rhizobium sp. & IAA & $22.40 \mu \mathrm{g} / \mathrm{ml}$ & \\
\hline Micrococcus luteus & IAA & $27.97 \mu \mathrm{g} / \mathrm{ml}$ & \\
\hline \multirow{2}{*}{$\begin{array}{l}\text { Bradyrhizobium japonicum } \\
\text { УКМ В-6035 }\end{array}$} & IAA & $\begin{array}{c}>680 \mu \mathrm{g} / 1 \\
(770 \mu \mathrm{g} / \mathrm{g} \mathrm{CD} \mathrm{B})\end{array}$ & $\begin{array}{l}\text { Leonova N.O. et al, } \\
2009,2010\end{array}$ \\
\hline & cytokinins & $\begin{array}{c}\sim 600 \mu \mathrm{g} / 1 \\
(670 \mu \mathrm{g} / \mathrm{g} \mathrm{CDB})\end{array}$ & \\
\hline $\begin{array}{l}\text { Bradyrhizobium japonicum } \\
\text { B-6018 }\end{array}$ & cytokinins & $1550 \mu \mathrm{g} / \mathrm{g}$ CDB & $\begin{array}{l}\text { Leonova N.O., } \\
\text { Bilyavska L.O., } 2010\end{array}$ \\
\hline
\end{tabular}

*CDB - completely dry biomass

receive stable and predictable results using phytohormonal substances (or their producers, microorganisms) it is important to do the correct dose calculation of the phytohormone 
applied exogenously, which requires the prior estimation of endogenous status of the treated plants. The problem with plant hormones application is even more complicated since at exogenous application the hormonal content is not simply added to the endogenous pool but also activates processes aimed on its utilization and possible changes of synthesis rates and disintegration of other hormones [32].

In general, there is no doubt that most of agronomically useful microorganisms benefit not only because of their capability of actively fix atmospheric nitrogen or mineralize hard-accessible phosphorus compounds, but also for their ability to produce biologically active compounds, including plant hormones. Therefore, the need for research of microorganisms-producers of substances of phytohormonal nature that may be potential bio-agents for microbial preparations of plant growth promoting action is obvious. Such studies will lead to the development of effective methods of optimization of plant hormonal status, which, in turn, might positively affect the production process of agricultural crops [49].

Reception simplicity of natural phytohormones of microbial origin, their relatively low cost, ability to detoxify within the plants, bounding in plant cells and their catabolization are among the main challenges of their use in crop production. However, phytohormones production in vitro does not mean that microorganisms will be able to synthesize them in natural conditions and their absorption can significantly affect the level of hormones in the plant. Therefore, when studying microorganisms-producers of phytohormones, in order to determine the effect of inoculation on plants hormonal content it is important to investigate the changes in hormonal content within the plants [50].

There are many unsolved problems associated with the use of phytohormones of microbial nature in agriculture. Therefore, research in this area continues and requires advanced determination methods of hormonal content in plants and exogenous phytohormons produced by microorganisms and can be potentially used by plants. The need for such research methods is determined by fact that the effect of plant hormonal substances of the plant primarily depends on their concentration and the concentration levels ratio of other phytohormones.

Phytohormonal research is one of the complex scientific problems that require considerable time and resources. At-first, that's due to the fact that plant hormones accumulate (both by plants and microorganisms) in a composite multicomponent complexes generally containing highly labile substances. Pools of any metabolites (including phytohormones) is a dynamic balance between input, output and intermediate pool. By increasing the pool size can result in both increased synthesis and reduced substances use at their constant intake. Conversely, low metabolite can equally result in low synthesis or very rapid transition in the inactive form [51].

Despite nearly a century of phytohormonal research there is still no clearly regulated methods of their quantitative determination that would be artifacts free. Lack of reliable and accessible laboratory system for content determination of these substances - is a global problem for scientists working in the field of agricultural microbiology. Such system is required for selection of promising strains - producers of plant hormones, for quality control of microbial preparations and biocomposts for phytohormonal content as 
well as for the determination of phytohormones in different types of soils, depending on the action of biotic and abiotic factors. Although plant hormonal analyzes remain a bottleneck in plant physiology and soil microbiology through trace concentrations of these substances and the presence of complex components in the analyzed material, the significant progress in the development of methods for extraction, purification and identification of phytohormones has been made in recent years.

Among the demands put forth today to analytical methods for phytohormones determination, the following are of the main importance: specificity and determination selectivity of single phytohormone or group of plant hormones; sensitivity, that would allow determination of phytohormones in picograms, and efficiency.

Determination methods of natural growth regulators have been rapidly improved over the past decades. If in 70's of last century, scientists were armed with simple and accessible, but not perfect bioassay methods, today modern precise methods that require complex and expensive equipment are used in many scientific centers. Nowadays the methodology choice is determined by the research challenges and technical capabilities of the laboratory. For example, a comparative assessment of the contents of phytohormone's class in experiment can be achieved by specific bioassays. However, the identification of certain phytohormones within the certain class, determination of their quantitative contents typically requires more complex instrumental methods.

The general scheme of phytohormones definition includes the following steps:

- sampling;

- phytohormones extraction from analyzed samples;

- separation of plant hormones from related impurities;

- qualitative identification and quantitative determination [52].

An objective assessment of phytohormone content in the tested material depends not only on the choice of sufficiently sensitive and selective analytical method, but above all, is determined by the representativeness of the selected sample. The full attention should be given to sampling procedures, due to the high cost of analysis: including cost of reagents, equipment depreciation and labor costs of qualified specialists.

Material selected for analyze should be fixed since phytohormones belong to the volatile compounds and at materials storage can be metabolized. Because of this, if necessary, at material storage (even for a short period) the probe fixation is used. Then fixed material is used for the extraction of hormones. One of the best methods of fixation is sample treatment with liquid nitrogen, followed by dry freezing. Dried fixed material can be stored without significant changes of phytohormonal status during the year, being placed in a desiccator and refrigerator with temperatures below $0{ }^{\circ} \mathrm{C}$. Samples fixed in liquid nitrogen, without freeze-drying can be stored for months at a temperature below $18{ }^{\circ} \mathrm{C}[53]$.

The disadvantage of most methods used for the quantitative determination of the plant hormones content is the fact that they require prior high quality and multiple purification of samples from impurities, while all known cleaning methods usually lead to losses of phytohormones enough as they are available in low concentrations.

Determination means of plants hormones contents can be divided into 3 groups: 
bioassays, physic-chemical and immunological (Figure). Considering the advantages and disadvantages of existing analytical methods, researchers can choose among them the one that is more acceptable to the specific research goals.

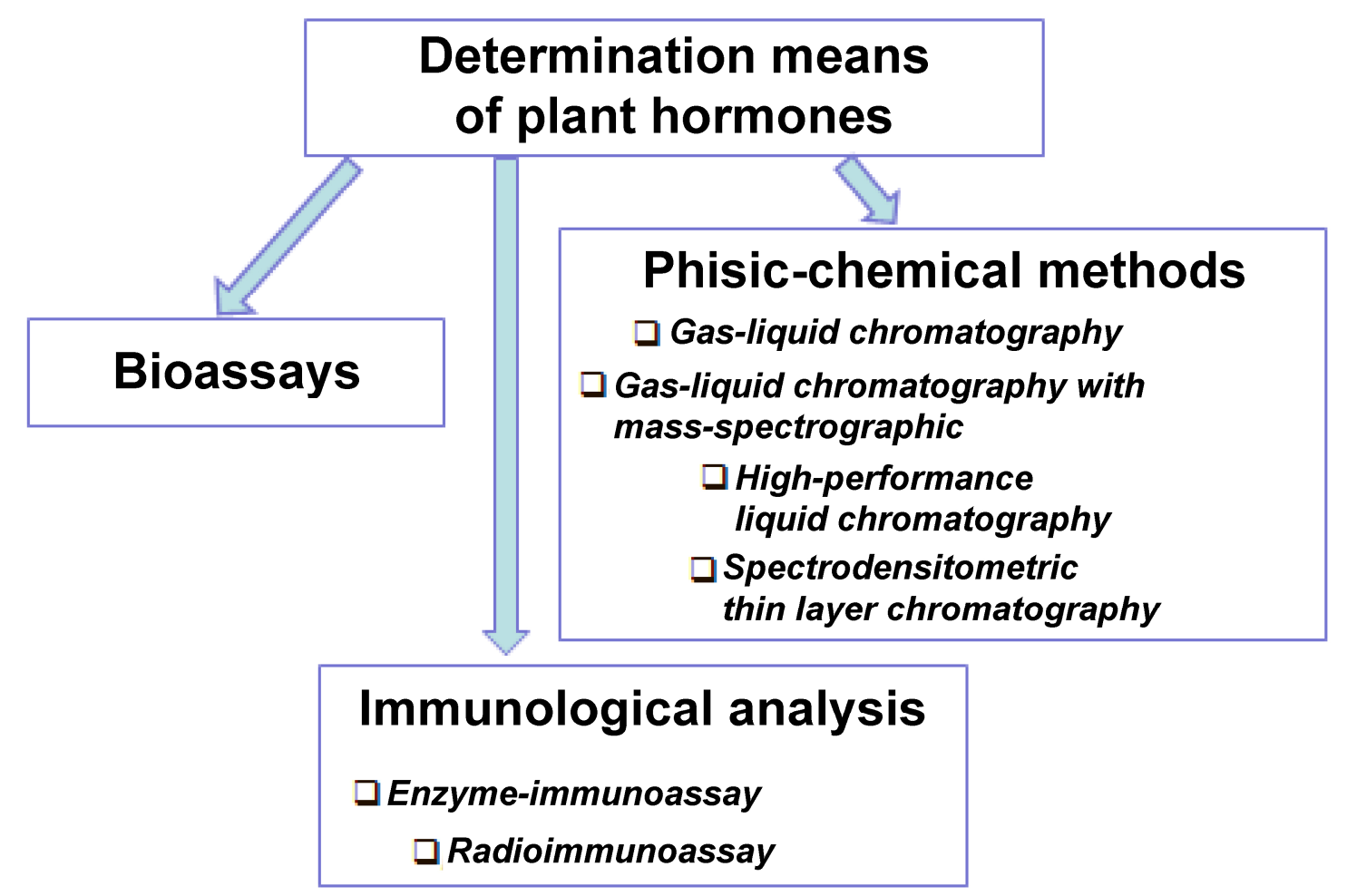

Fig. Methods for used determination the content of phytohormones

Bioassays [54 - 62] - routine methods based on the radical properties of hormones, which in contact with sensitive plants or isolated plant organs generate the response (growth acceleration, accumulation of pigments, etc.) correlated with the amount of the available hormone. The tested plant extract or bacteria culture is put in contact with the plant while the test response is compared to the hormone standards, which results in the estimation of the phytohormonal content in the sample. Bioassay objects should meet the following requirements: 1) standardization of source material and its responses to phytohormones; 2) high sensitivity to the tested substances - test object should respond to the small concentration of plant hormone, which is possible only at low initial concentration of substances in the cells; 3 ) specificity (reacts only for a certain group of phytohormones); 4) reproducibility of results. By the nature of physiological responses to phytohormones bioassays are divided into growth assays (bending and extension of coleoptiles of cereal crops), pigment assays (synthesis of chlorophyll and carotenoids) and enzyme assays (synthesis of enzymes, e.g. $\alpha$-amylase). When compared to the physicchemical methods the productivity of bioassays is much higher, but bioassay experiments last for not less than a day or even longer. For example, in tissue culture (bioassay for cytokinins) the results will be available in about a month. Finally, the significant disadvantage of bioassay methods is that their repeatability is quite uneven, since the 
researcher in this analysis deals with plants or their organs and tissues that are complex biological systems which are very difficult to standardize. In addition, bioassay methods in fact are semi-quantitative and they often play a supporting role in the detection of phytohormones. Bioassays provide researchers very first information about the presence of these substances in the plant. They are widely used on initial screening for new hormones and at final stages of analytical work related to the physiological characteristics of the detected phytohormones [63]. The undoubted advantage of bioassay methods is their minimal equipment requirements. Among disadvantages are overestimation of actual content of phytohormones through the expression of hormonal activity of certain classes of secondary metabolites of plants and microorganisms.

During last $30-40$ years our knowledge of plant hormones had increased due to extremely powerful analytical capabilities of new equipment. The sensitivity of modern methods is so high that a small amount of a substance can be easily detected and identified. However, all these instrumental methods based on well-known and relatively simple physical and chemical laws.

Analyzed samples containing plant hormones are mixtures. Even when using highly effective ways to extract certain compounds from the sample we still have to analyze the mixture. Therefore, the prerequisite for successful study of natural phytohormones is a thorough preparation of material for analysis. Analyzed extracts usually contain not only phytohormones but also different compounds that can interfere with the determination of hormones. Chromatography is used for separation of phytohormones from impurities which is based on the differential distribution of multiple mixtures of substances between two phases (mobile and fixed) that do not mix with each other.

Obviously, one of the best methods that can be used in plant hormonesresearch is high performance liquid chromatography (HPLC) [64-66]. This type of chromatography is now often used in the study of complex mixtures of substances (which include both plant extracts and microbial culture medium). Its popularity is due to the method versatility, since it can be used to separate almost any (except macromolecular) compounds. Sample analysis process takes place in two stages: sample separation on composite components using chromatographic column and detecting and quantification of the content of each component.

High performance thin layer chromatography (up-to-date TLC) [67-68] uses completely automatic scanning of TLC plates. The principle of modern scanning densitometer is based on the detection of areas of separated fractions of applied on chromatographic plate mixture using stationary light beam in the visible or UV range at wavelengths of 254 and $365 \mathrm{~nm}$. The relative distance between the detected areas and start line is used for characterization of the chemical nature of the analyzed substances while the areas intensity - their quantity.

Mass spectrometry - is modern analytical method characterized with high sensitivity, reliability and informational capacity [69]. Recently, mass spectrometry has experienced significant technology upgrade that allows its application for determination of various biologically active molecules. Mass spectrometer allows determination of molecule mass by measuring the ratio of mass to the charge its ion $(\mathrm{m} / \mathrm{z})$. At molecules 
ionization generated ions are electrostatically directed into the mass analyzer, where they are differentiated according their $\mathrm{m} / \mathrm{z}$ and detected. The result of molecules ionization, separation and detection of ions is a spectrum, which can used for determination of molecular weight and receipt of certain information about the structure of matter and perform its identification.

Nowadays, analysis of phytohormonal content is widely used in combination of mass spectrometry with various chromatographic methods [70-74]. Chromatography-mass spectrometry is a hybrid method with two independent of each other processes separation and analysis. Combining chromatograph with mass spectrometer facilitates the interpretation of the spectra, since prior to the bombing mixture is separated into individual components. Also mass spectrometer is the best detector for chromatography. Direct connection of these two devices into the single chromatography-mass spectrometry system leads to the significant increase of each device capabilities. Chromatography-mass spectrometry is a multiplex method used for the determination of phytohormones in the sample, allowing monitoring the pool of phytohormones in real time. Modern mass spectrometers are equipped with computer programs that include banks of mass spectral data used for the substances identification. The main disadvantage of this method, like many others, is the need to perform complex sample preparation [75-78].

Unfortunately, HPLC, spectrodensitometric method and chromatography-mass spectrometry are not widely available in conventional biological laboratories because of their high cost. The common feature for most methods is the complexity of phytohormones determination and duration of the analysis. Despite the obvious advantages of physical and chemical methods and their indispensability in some cases, we should highlight that they are difficult to use if needed to maintain quick control of the phytohormones' content. The high complexity of these methods does not allow quickly analysis of large numbers of samples, which clearly demonstrated the urgent need for rapid express quantification methods of plant growth regulators.

The basis of immunochemical methods is formed on the specific antibody interaction of relevant substances that act in this system as antigens. Antibodies are generated in animals in response to the introduction of foreign substances, ie immunization. The specificity of antibodies allows them to "recognize" the relevant antigen and quantitatively interact with them to form antigen-antibody complex.

Radioimmunoassay analysis (RIA) is quantitative method used for determination of phytohormones in biological fluids based on competitive binding of stable and similar to them radionuclide-labeled substances with specific binding systems, followed by their detection at radiospectrometer [79-82]. Iodine isotope is most commonly used for labeling of antibodies or antigens. RIA also foresees the use of expensive equipment, such as gamma counters. Moreover, radioactive materials used for the analysis, have a relatively short shelf life of diagnostic kits due to the breakdown of the radioactive label. These features of radioimmunoassay analysis are considered as its main disadvantages that result in gradual method replacement with the enzyme immunoassay modifications that can achieve the sensitivity of RIA without the use of radioactive isotopes.

In our opinion, the method of enzyme-linked immunosorbent assay (ELISA) meets 
the rapidity and simplicity requirements. Phytohormones are not the only class of biologically active compounds, which are determined using immunochemical methods. For example, immunochemical test systems used for determination of hormones in animals and humans have been developed long time ago and being already producedon industrial scale. However, for many years they have remained beyond the interests of researchers interested in plant hormones studies, since phytohormones by their nature a haptens, or incomplete immunogens, which are unable to induce an immune response in animals. However, in the early 80 's of the last century the receipt of immune serum to auxin, cytokinin, gibberellin and ABA has been described.

Today the improving process of the immune phytohormones testing methods continues [83-96]. Over the time, the thorough restructuring of the entire analytical system is performed, but the basis of the method remains the same:

- receipt of phytohormone conjugate with protein or other carrier to give it the properties of the antigen;

- receipt of immune antisera for this conjugate and its use for the determination of phytohormone in appropriate ways [90].

The indirect solid phase ELISA-based method is used for determination of phytohormones content. It is based on the competition between the analyzed plant hormone and antigen (phytohormone-protein conjugate) immobilized on polystyrene surface for the antibody binding sites. After the establishment of equilibrium in the system and separation of the components of immunochemical reaction the antigen-antibody complex formed on the surface of the tablet is detected by with antispecific antibodies conjugated with the enzyme molecule (horseradish peroxidase). At this the intensity of chromophore response of enzyme marker is proportional to the concentration of immobilized antibodies and inversely proportional to the content of phytohormonecompetitor in the test solution.

For a long time the use of test systems developed for the determination of phytohormones, despite the known specificity of immunoassays as such, had included the preliminary multi-stage samples purification. Today upon the creation of test systems researchers are looking for the opportunities that will allow skipping the difficult samples cleaning stages. For example, the employees of the Department of Plant Physiology of Moscow State University had developed two systems for immunochemical determination of indolyl-3-acetic acid, which parallel use allows quantitative determination of free and bounded IAA forms without methylation stage and sample fractionation [97, 98].

It is expected that the use of new and more sophisticated quantitative analytical methods for determining the content of plant hormones will help researchers to answer more questions about phytohormones of plants and microorganisms that have not being answered yet. 